\title{
MICROPROPAGATION OF HERITAGE RHODODENDRON COLLECTIONS AT THE ROYAL BOTANIC GARDEN EDINBURGH
}

\author{
Neil Davidson ${ }^{l}$
}

\begin{abstract}
The most recent efforts at micropropagation of Rhododendron species started at the Royal Botanic Garden Edinburgh in 2013. This paper outlines the methods and practices adopted, and highlights some of the problems and pitfalls encountered throughout the process. At the close of 2017 the first plants propagated using in vitro techniques were planted at Benmore Botanic Garden in Argyll, Scotland.
\end{abstract}

\section{INTRODUCTION}

In recent decades many of the important heritage rhododendrons in the living collections of the Royal Botanic Garden Edinburgh (RBGE), some of which were collected by famous plant collectors including George Forrest, Joseph Rock and Ernest Henry Wilson, have been in steep decline (Fig. 1). There are several reasons for this decline. Heavy infestations of cushion scale (Pulvinaria floccifera), a sap-sucking insect causing black sooty mould, has weakened some individuals. Several Rhododendron species are not suited to Edinburgh's climate, which can have long, dry spells, as many of them naturally occur in much wetter climates. A major contributor to decline is the age of the collection; RBGE has been growing and researching rhododendrons since the late 19th century.

In May 2012, Neil Davidson and Ross Irvine, Senior Horticulturist at RBGE, attended a three-day training course on micropropagation at Duchy College in Cornwall. The course, run by Ros Smith, provided a summary of the techniques and skills essential for adopting a micropropagation programme for Rhododendron species at RBGE. Ros has been heavily involved in the conservation of rare and heritage plant collections using micropropagation procedures. She has propagated material from many of Cornwall's heritage gardens, particularly since the spread of Phytophthora ramorum and Phytophthora kernoviae into the south-western regions of the UK.

A key element of successful micropropagation is the exclusion of external contaminants. In 2008, Ros Smith and Barry Mulholland published a paper comparing the in vitro treatment of floral buds with vegetative shoots to examine which of these enabled this exclusion most successfully (Smith \& Mulholland, 2008). Their results found that floral buds were the most reliable method for keeping clean cultures, with a 65 per cent

1. Neil Davidson is a Horticulturist at the Royal Botanic Garden Edinburgh. Address: 20A Inverleith Row, Edinburgh EH3 5LR, UK.

Email: ndavidson@rbge.org.uk 
Fig. 1 Rhododendron species in decline and losing vigour at RBGE. Photo: N. Davidson.

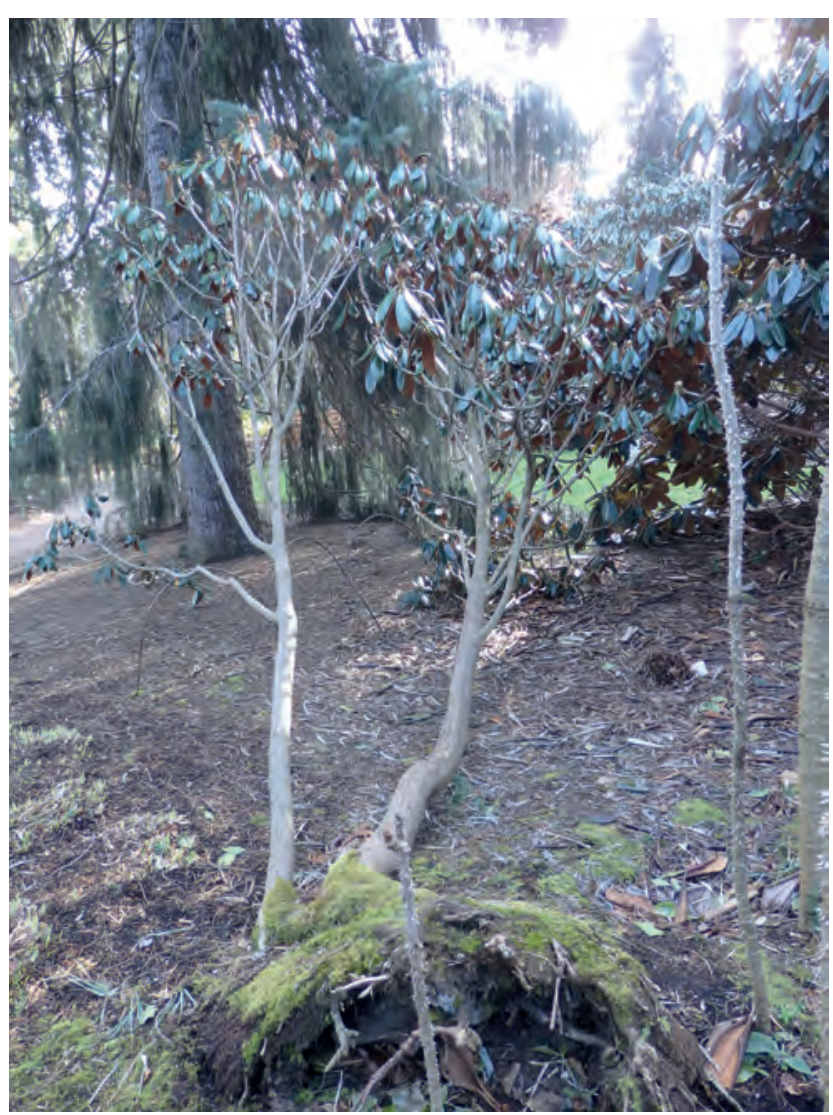

success rate. It is this method, using rhododendron flower buds, that we have adopted at RBGE.

THE ADVANTAGES AND DISADVANTAGES OF MICROPROPAGATION

Micropropagation boasts some advantages over more traditional forms of propagation. One major benefit is freedom from disease and viruses as sterile techniques are in place throughout. This is particularly important since Phytophthora ramorum has been discovered on the Ericaceae collection at Edinburgh, Logan and Benmore Botanic Gardens. A second benefit is vastly improved vigour when compared to traditional propagation methods such as cuttings. An old collection often means that plants reared by these methods are sometimes leggy, weak and lack vigour. Quality cutting material is seldom available from a declining plant; this will further reduce any chance of obtaining a high-quality propagule. A third benefit is the number of propagules which can be raised: whereas one cutting potentially gives us one plant, with micropropagation one floret could give rise to many plants. Finally, as a survival strategy, plants in poor 
condition often flower prolifically; this means that on even the poorest of specimens there is often an abundance of flower buds for us to use.

There are, of course, some downsides: most notably time constraints on staff, and higher skill levels and technical ability required. Keeping clean cultures from the beginning was the first hurdle to overcome. This was largely achieved by practising sound laboratory techniques and by comparing different sterilising and cleaning methods - essentially learning by trial and error. We have been aided by hints and tips from other laboratory users over time; it is always helpful to receive information about successful working practices. Some rhododendrons have sticky buds, and even after the most thorough cleaning process external contamination persists (Fig. 2). Currently the only response to this seems to be to process more florets. The browning of buds is also still a problem in some cases. During this project we have found that there is always more to learn.

\section{SPECIES SELECTION AND BUD COLLECTION}

It would be a herculean task to attempt micropropagation of the entirety of RBGE's rhododendron collection, so prioritisation is necessary. Plants chosen for inclusion in the project are of heritage value, wild collected, rare or unusual cultivars or species where only a few individuals remain. Priority is also given to larger-leaved species as these do not propagate readily from cuttings.

Floral buds are collected between the months of December and February shortly before flowering. Six to eight buds are taken from each specimen, and leaves are removed leaving only the stem and the bud itself. These are placed into a sandwich bag along with a label containing the accession number and qualifier of the individual. These are stored in the fridge at $4^{\circ} \mathrm{C}$ until they are processed, a maximum of two weeks after collection.

\section{PROCESSING THE BUDS}

In the laboratory the first step is to remove the outer scales of each bud; these hold the most contaminants and are discarded (Fig. 2). Each bud is treated with a 70:30 thin bleach: distilled water mixture using a toothbrush to clean the outer part of the bud, and then rinsed thoroughly with distilled water.

The stems are then removed, and the buds are placed into a 30:70 thin bleach: distilled water mixture and left to soak for 20 minutes. The solution is less concentrated at this stage because the buds are left in it for some time and a stronger solution could damage them. They are then triple rinsed in sterilised distilled water (distilled water that has been through an autoclave) and placed in the laminar flow cabinet ready for processing.

The laminar flow simply decreases the chances of airborne external contaminants infecting our explant material by circulating filtered air towards the user. Working inside 


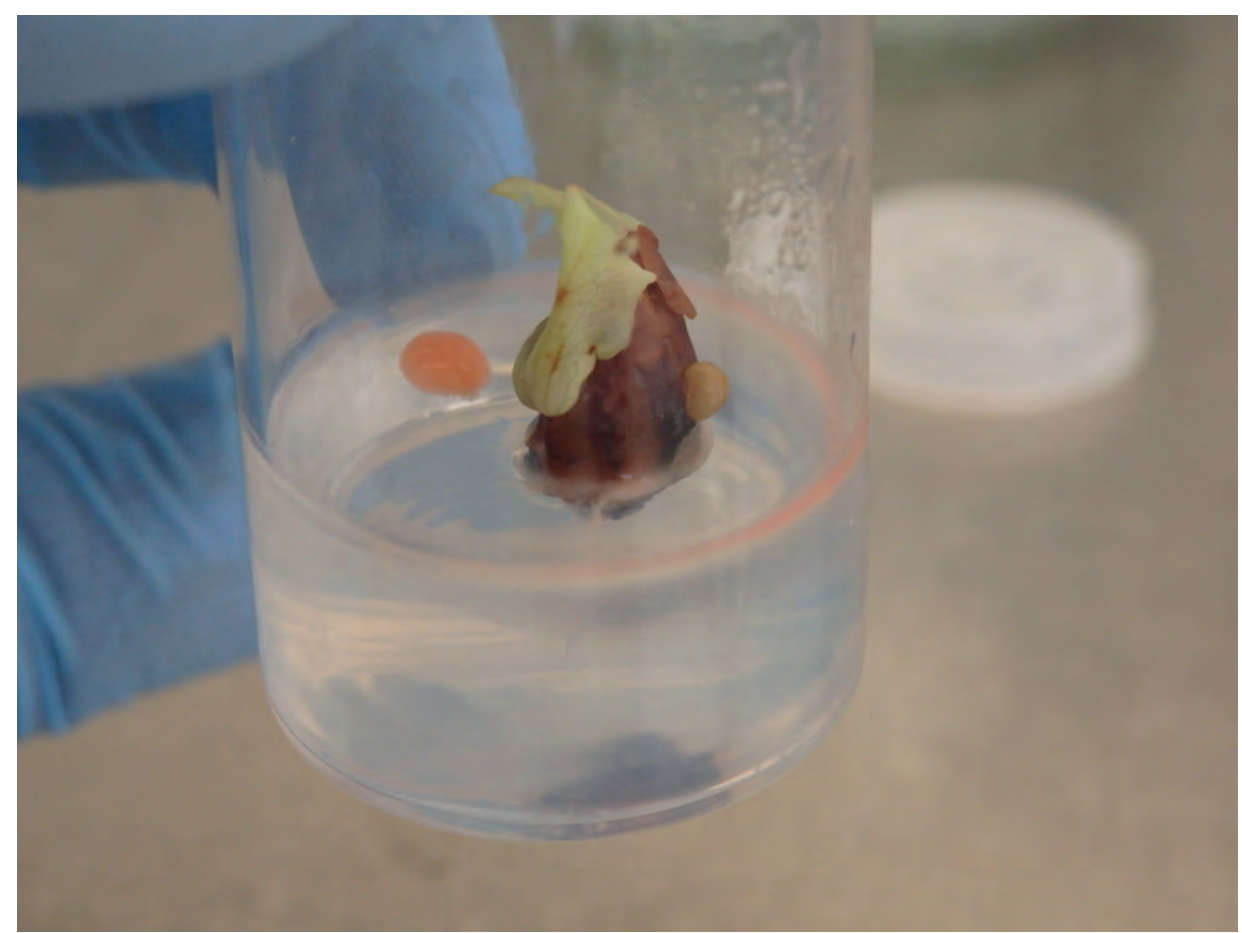

Fig. 2 The orange substance on the surface of the agar are contaminants not killed during the cleaning process on Rhododendron grande, in this case. Photo: N. Davidson.

it (Fig. 4) the outer bud scales are removed until the first floret is exposed. The largerleaved species often have large, fat flower buds and may contain up to 20 florets. The florets are removed with a sterilised scalpel, placed in an antioxidant mixture containing ascorbic and citric acid alongside plant growth regulator thidiazuron (TDZ) and left to soak for 15 minutes. This antioxidant mixture helps to prevent rapid browning of the florets and the TDZ acts as a catalyst for the callusing process (Fig. 5).

\section{BUD MAINTENANCE AND DEVELOPMENT}

When the florets have been removed they are placed into a vial of agar medium. In the initial stages of development, a percentage of florets are likely to contain external contaminants not killed in the cleaning process, so only one bud is placed in each vial, preventing one contaminated floret from infecting others. Vials are labelled and then placed in a growth cabinet set at $22^{\circ} \mathrm{C}$ with 16 hours of light. The florets are put into fresh agar medium every one to two weeks because nutrients and hormones become depleted after this time. Once the level of contaminants contained within the florets has subsided they are placed five to a jar. Subsequent changes into fresh agar medium are required every four to six weeks to sustain optimum development. 


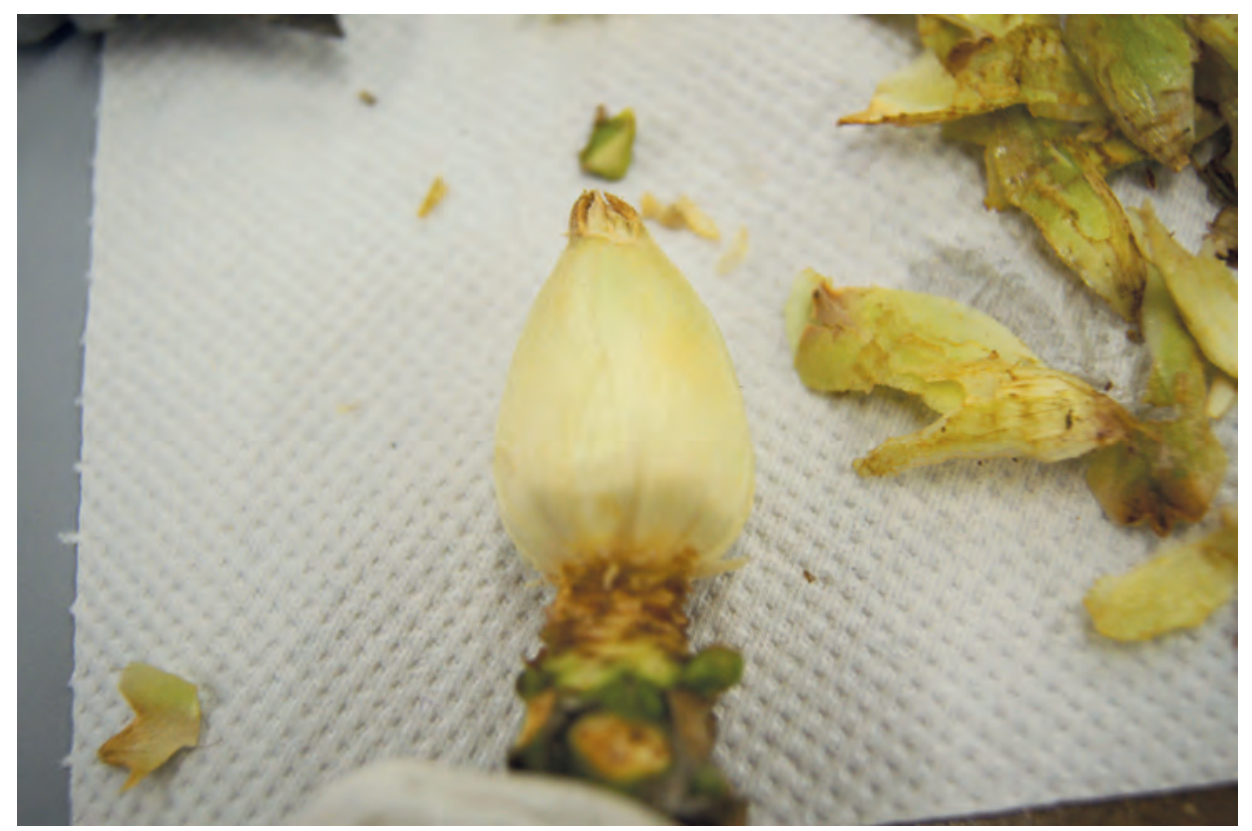

Fig. 3 Outer bud scales are removed to reduce contamination. Photo: N. Davidson.

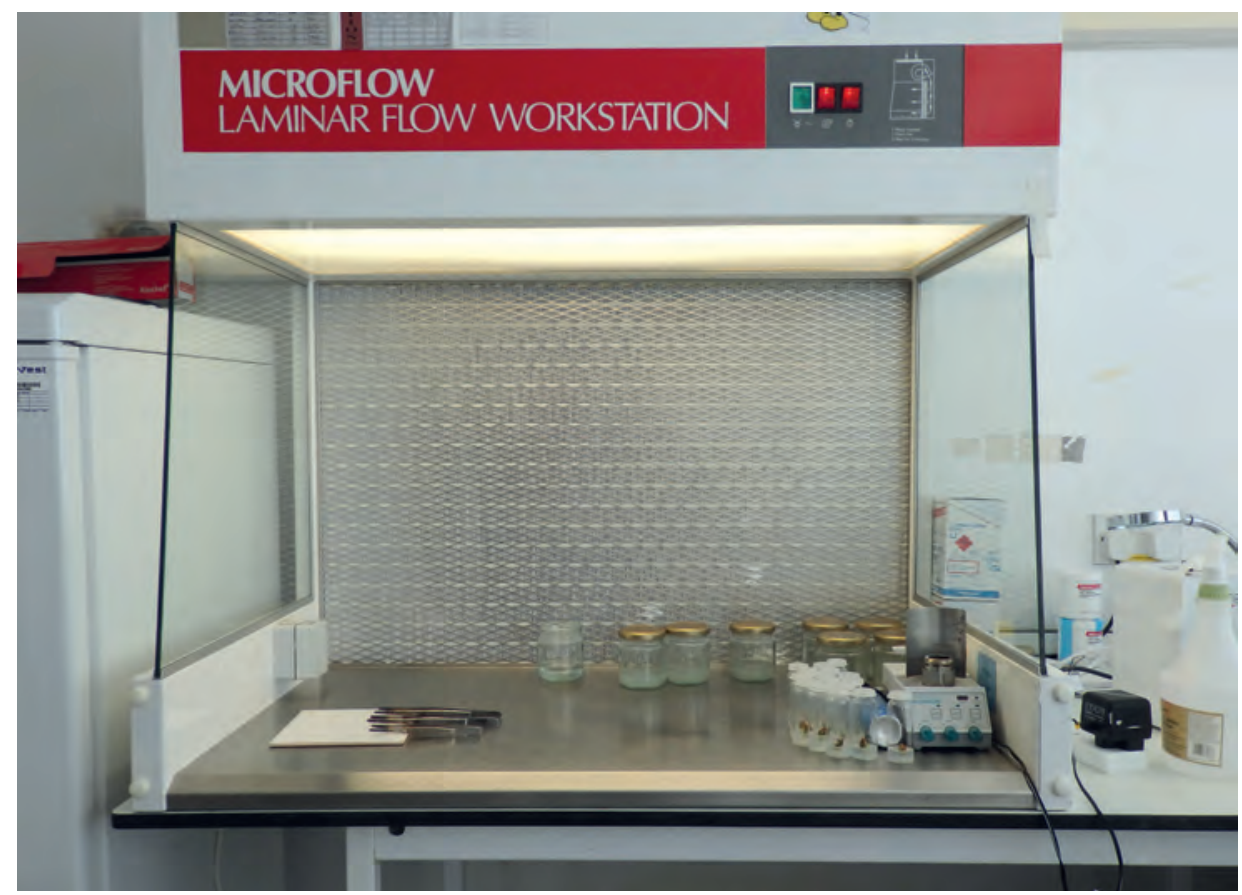

Fig. 4 Laminar flow workstation: florets are moved into fresh agar medium in the sterile environment. Photo: N. Davidson. 


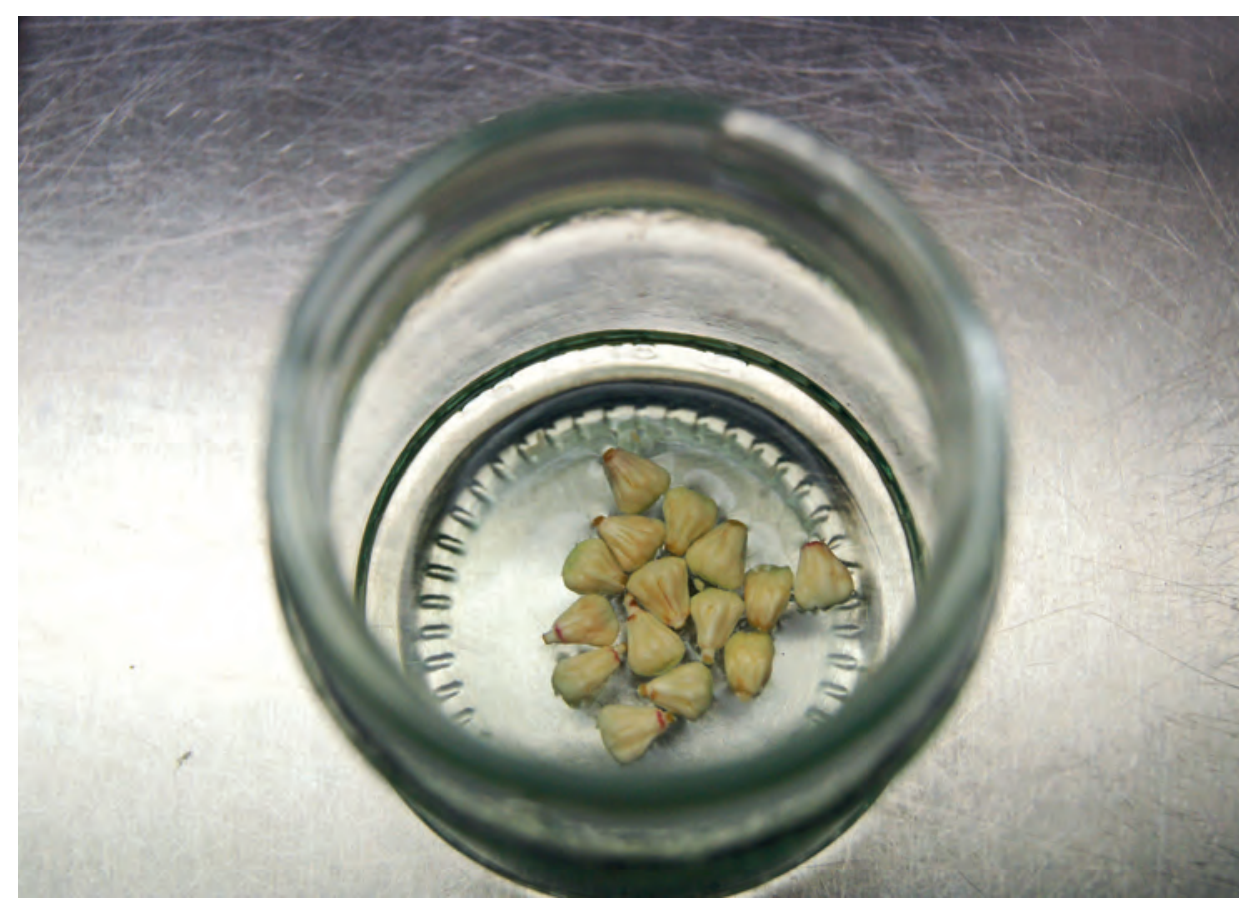

Fig. 5 Florets soaking in antioxidant to prevent browning of the tissue. Photo: N. Davidson.

About six to eight weeks after insertion callusing will occur (Fig. 6). After four to six months (sometimes longer) the explant will have sprouted leaves, which look like miniature bushes (Fig. 7). Eventually these shoots will elongate. Cuttings are taken from these shoots and are inserted into a rooting agar (Fig. 8). Two to three months later rooted cuttings will be ready for potting into a regular growing medium. The nutrient agar is washed off and the explants are potted into a mixture of propagation bark, charcoal and controlled-release fertiliser. To acclimatise them effectively they are weaned in a growth room at $18-20^{\circ} \mathrm{C}$ with 16 hours of light. Thereafter they are placed into the Temperate Glasshouse (Fig. 9).

\section{AGAR MEDIUM}

Florets are put in an agar medium of nutrients and hormones. For Rhododendron species there are three stages of growth and three corresponding agar mixes: initiation, multiplication and rooting. Here the individual components in the agar medium for these three stages are briefly outlined along with the reasons for their use.

Agar: Extracted from several species of red algae, this is the setting agent that holds all ingredients.

McCowan's Woody Plant Basal Salt Mixture: The fertiliser component, this contains all the macro and micro nutrients essential for growth and development. It should be 

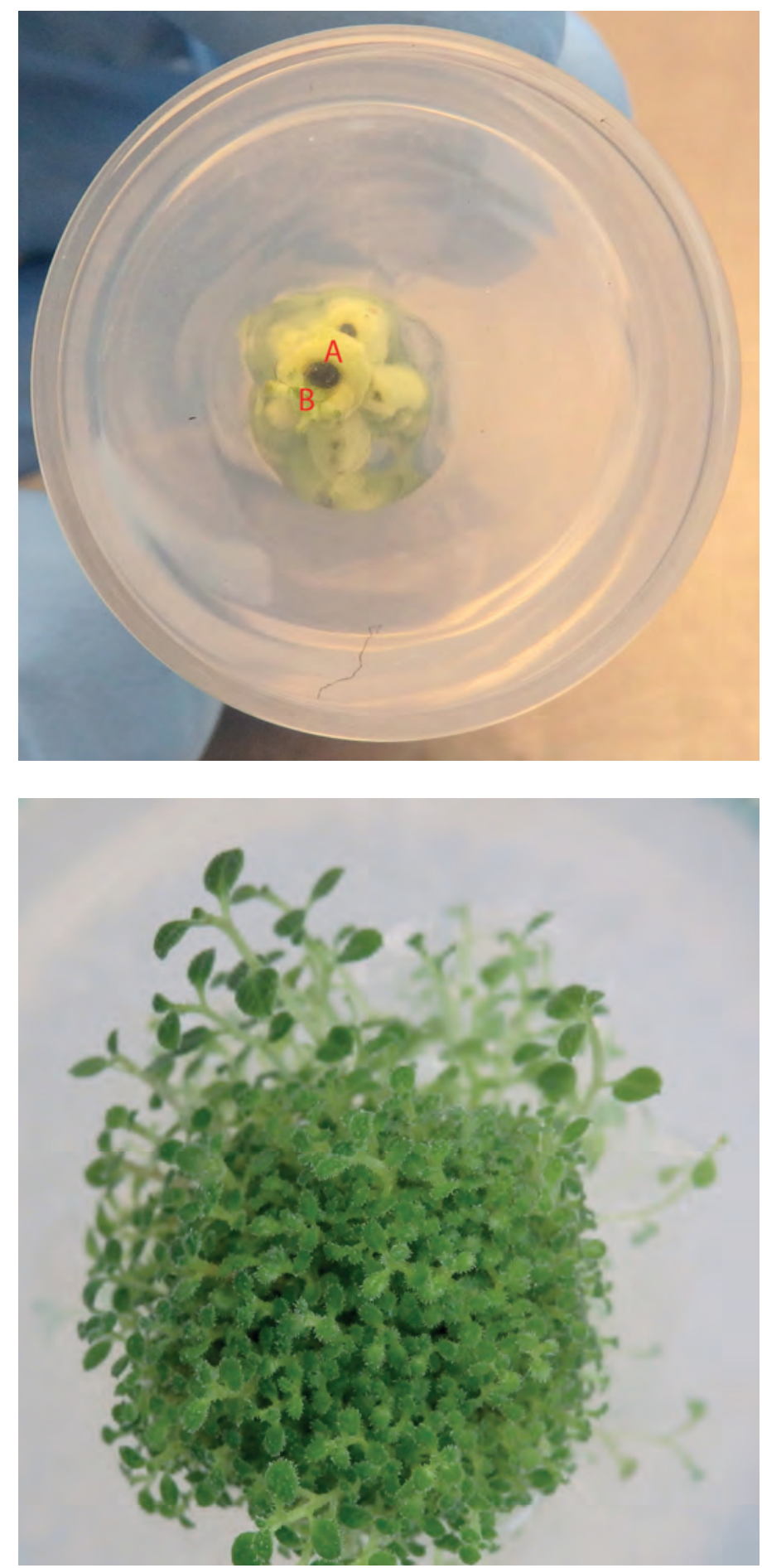

Fig. 6 This

Rhododendron taliense in stage 1 agar medium shows callus swelling on pedicel. A: Callusing around pedicel.

B: Shoot initiation; cells have specialised into photosynthetic cells (green can be seen). Photo: N. Davidson.
Fig. 7 'Miniature bushes'. This Rhododendron barbatum in stage 2 agar medium measures approximately $5 \mathrm{~cm}$ across. The next step will be to take cuttings from these shoots and insert them into stage 3 rooting medium. Photo: N. Davidson. 


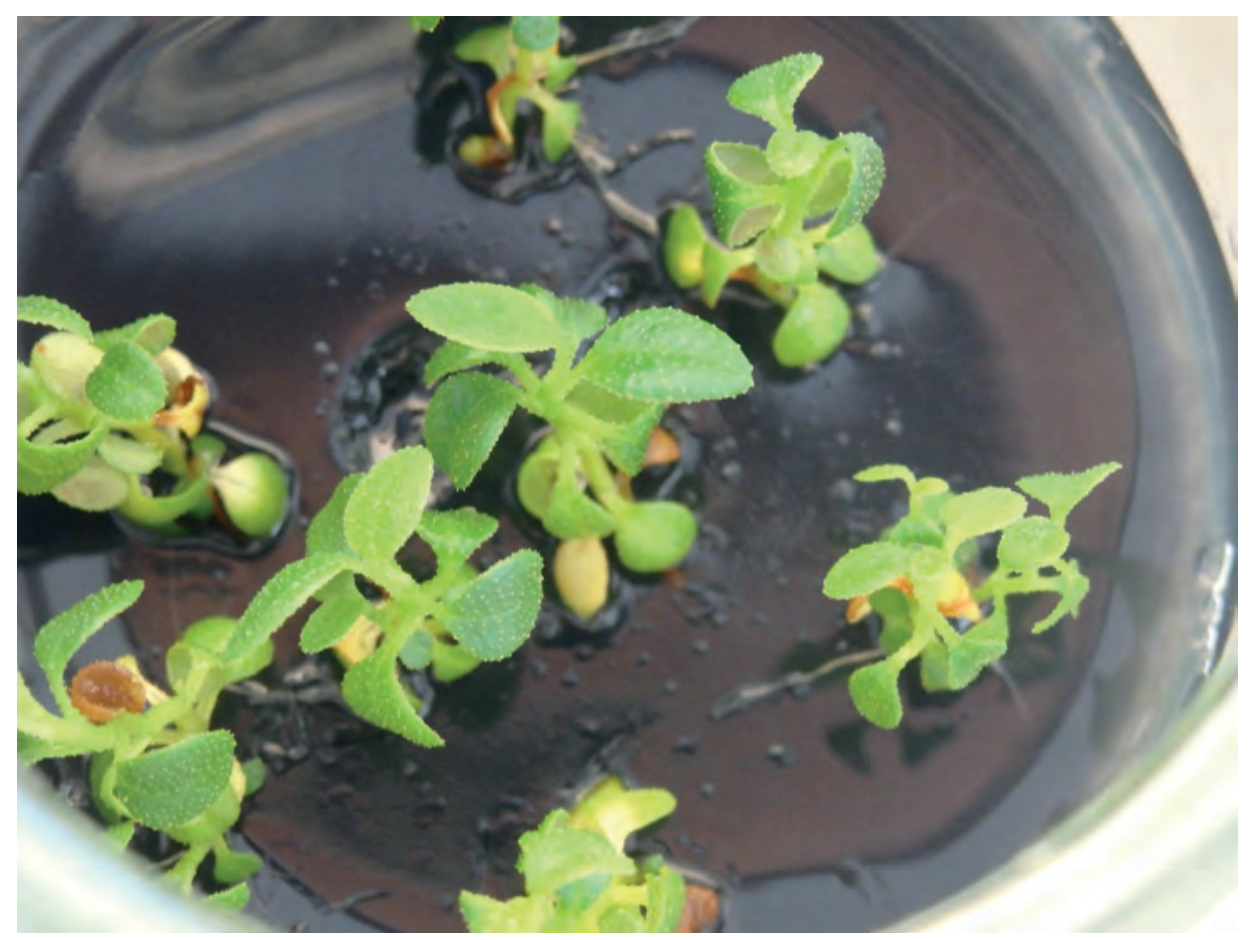

Fig. 8 Rooted cuttings of Rhododendron calophytum var. calophytum in stage 3 agar medium. The dark hue is from charcoal added to the rooting phase medium. Hormone IBA (indole butyric acid) is used to promote rooting. These are ready to be potted into a growing substrate. Photo: N. Davidson.

noted that Anderson's Basal Salt Mixture is traditionally used for rhododendrons, but this is less widely available.

Ferrous sulfate and ethylenediaminetetraacetic acid (EDTA): Added together, EDTA allows ferrous sulfate (one of the macro/micro nutrients) to be more easily accessible to the cultures.

Thiamine Hcl: This B vitamin plays a vital role in the citric acid cycle which is an important process in cell respiration. It is therefore critical for growth.

Inositol: Another B vitamin that aids cell growth.

Sucrose: This is a product of photosynthesis, but cultures in vitro will not be able to produce all the sucrose they need. It is added in the form of cane sugar to provide energy.

Activator charcoal: Charcoal is often used in horticultural substrates as a filter to remove impurities and toxins that inhibit root growth.

\section{GROWTH REGULATORS}

Over the course of the three stages, four growth regulators, or hormones, are added to the agar medium. These are: 


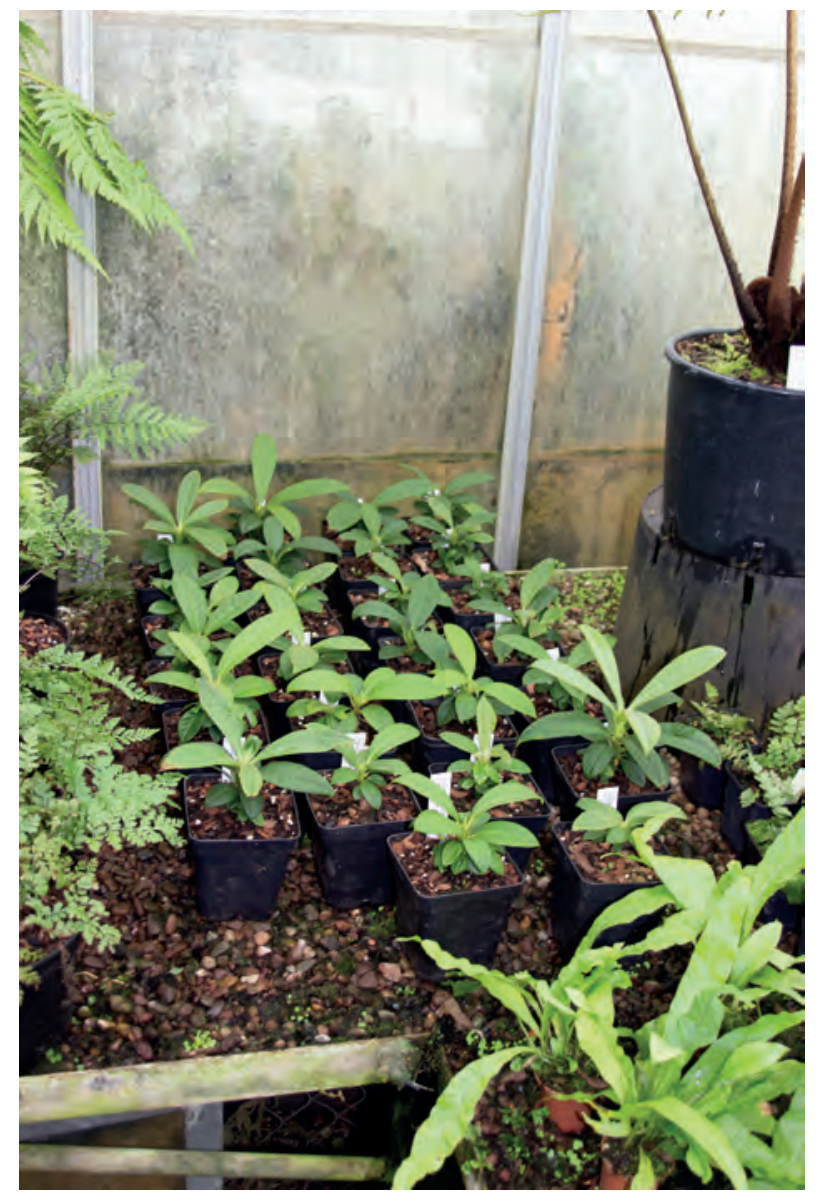

Fig. 9 Rhododendron calophytum var. calophytum growing in the Temperate Glasshouse to acclimatise before planting outdoors. Photo: N. Davidson.

\section{Auxins}

3-Indoleacetic acid (IAA): A naturally occurring auxin, sometimes used as a rooting hormone.

Indole-3-butyric acid (IBA): A synthetic auxin frequently found in commercially available rooting powders and gels. It is used in micropropagation as a catalyst for rooting.

\section{Cytokinins}

6-( $\gamma, \gamma$-Dimethylallylamino)purine $(2 i P)$ : A synthetic cytokinin used in our cultures to promote cell division and initiate shoot elongation.

Thidiazuron (TDZ): A plant growth regulator with cytokinin-like behaviour. It aids callusing in our cultures during the initial stages of development. 


\section{THE ROLE OF AUXIN AND CYTOKININ IN THE MEDIUM}

It is worth noting that auxins encourage cell enlargement and root initiation, while cytokinins generally initiate cell division and shoot growth. Hormones also interact with each other and with other chemicals, and are influenced by environmental factors such as light and temperature. It is by altering the amounts of auxin and cytokinin and their respective ratios in the agar that cultures can be stimulated to grow and develop in a particular fashion (Kyte \& Kleyn, 1996).

\section{PROBLEMS OCCURRING WITHIN THE MEDIUM}

On occasion, the agar medium is stained a blue-black colour, caused by bleeding from necrotic tissue in the cultures. This can occur when cuts are made with a blunt scalpel, if the cultures are old or if the medium is too runny from a lack of agar (Kyte \& Kleyn, 1996). This bleeding poisons the medium and will check the growth and development of the culture. Regular checking is essential and moving cultures to a new container will help alleviate this problem.

Rooting can occur in stage 2 (multiplication). Although unusual, it does happen with some older cultures. This could be caused by a problem with the auxin to cytokinin ratio. In this instance, a decrease in auxin or an increase in cytokinin may resolve the issue.

\section{DEVELOPMENTAL STAGES}

The ingredients described above are used in varying quantities in the agar medium at RBGE throughout the three stages. The different ingredients and quantities are explained here in order to illustrate how changes in the ingredients of the agar medium can manipulate the growth of the cultures.

\section{Stage 1: Initiation}

All the agar media during the three stages contain agar, basal salt mixture, EDTA, vitamins and sucrose. Most of the variation is in which growth regulator is added or removed. For the first two stages, after the ingredients have been added the $\mathrm{pH}$ value is set to 5.5.

To promote a rapid response from cultures in the critical preliminary stages, three growth regulators are used during stage 1: TDZ, $2 \mathrm{iP}$ and IAA. The goal for this first stage is to get cultures to callus, sprout leaves and then commence shoot elongation.

\section{Stage 2: Multiplication}

There is only one change in the agar medium for the second stage: TDZ is removed. As the cultures begin the process of shoot elongation they are moved to the multiplication 
medium. TDZ inhibits this shoot elongation, which is essential. It is in stage 3 that cuttings are taken from these shoots.

\section{Stage 3: Rooting}

Cuttings are taken in the laminar flow cabinet and placed in the stage 3 medium. Changes in the agar medium are significant here. The basal salt content is raised to accommodate the removal of nutrients directly from the agar medium by roots. The only growth hormone present is IBA, which is used to catalyse rooting. Activator charcoal is added to filter out impurities. The $\mathrm{pH}$ is adjusted to 5.2 for rooting.

\section{THE FUTURE}

During the autumn and winter months of 2017 the first rhododendron plants from micropropagated material were planted at Benmore Botanic Garden (Fig. 10), four years after the project began. It will be interesting to see how these establish and mature. The long-term goal is to distribute this material to other gardens throughout the UK, so that a genetic lineage is maintained. It is by doing this that we hope to safeguard the future of this collection.

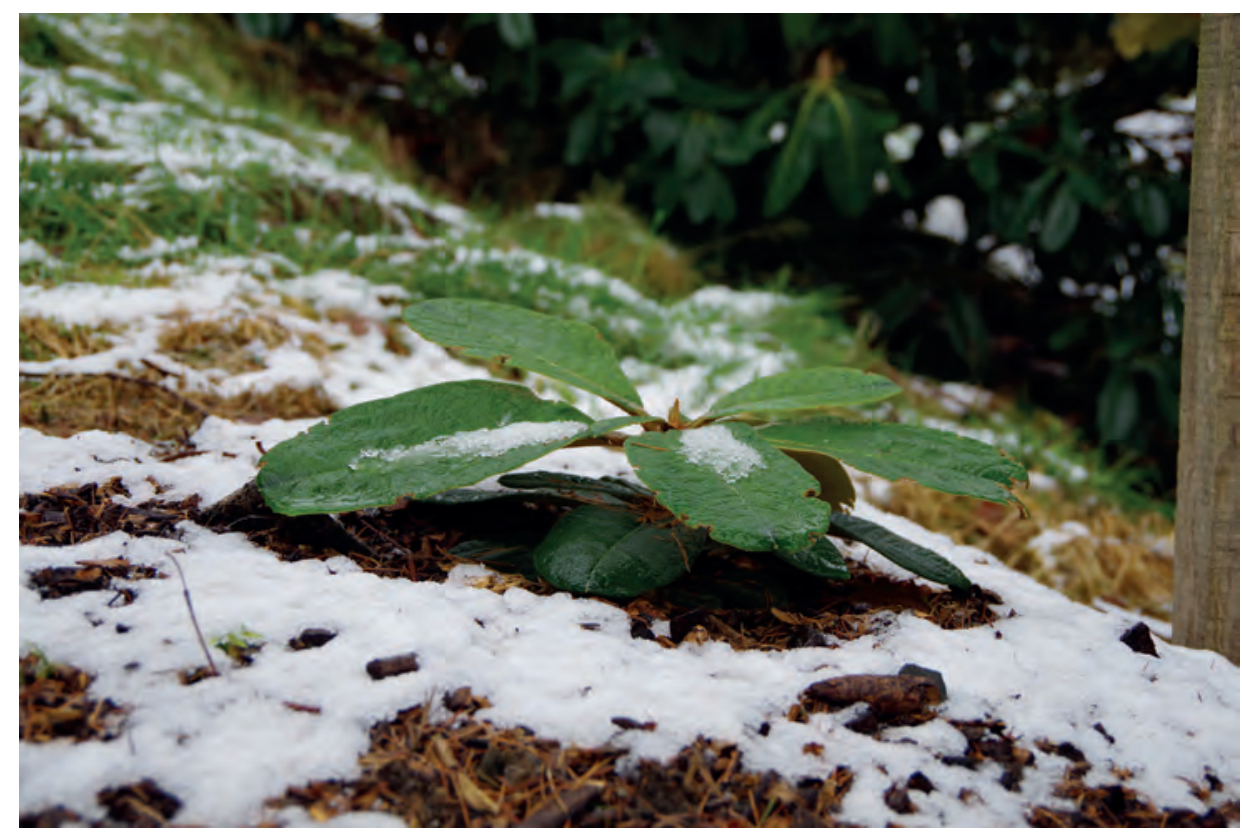

Fig. 10 Rhododendron arizelum planted at Benmore Botanic Garden in December 2017. Photo: N. McCheyne. 


\section{ACKNOWLEDGEMENTS}

Special thanks must go to Ros Smith from Duchy College; without her guidance, assistance and advice in the early days, we would have never reached this point. Thanks also to Ruth Hollands, who has provided much-needed help in procuring laboratory chemicals and equipment and given useful advice when needed.

\section{REFERENCES}

KYTE, L. \& KLEYN, J. (1996). Plants from Test Tubes. An Introduction to Micropropagation (3rd edn). Timber Press, Portland, OR.

SMITH, R. \& MULHOLLAND, B. (2008). Use of micropropagation for the conservation of rare Cornish garden plants at risk from $P$. ramorum. Combined Proceedings International Plants Propagators'Society, 58: 456-460. 


\title{
THE HISTORY OF THE WALLED (FORMAL) GARDEN AT BENMORE BOTANIC GARDEN
}

\author{
David Gray ${ }^{l}$
}

\begin{abstract}
ABSTR ACT
The 2.02 ha site containing the Category B listed Walled Garden at Benmore is currently the subject of a major redesign proposal and active fundraising programme. The purpose of this article is to raise the profile of the project by investigating and highlighting the historical development of the site. This retrospective study is also intended as a support to contemporary redevelopment plans and as a demonstration of how the past underpins and informs the future.
\end{abstract}

I am frankly and absolutely for a formal garden ... It is a small piece of ground enclosed by walls ... There is not the least attempt to imitate natural scenery (Phillpotts, 1906, p. 54).

\section{INTRODUCTION}

Benmore Botanic Garden is located on the Cowal peninsula within the district of Argyll and Bute on the west coast of Scotland. The 48.5 ha policies surrounding Benmore House have benefited from almost 200 years of tree and other ornamental planting. Successive generations of private landowners invested heavily to improve the appearance and productivity of the estate. The documented involvement of the Royal Botanic Garden Edinburgh (RBGE) at Benmore commenced in 1925 (Anon., 1925b).

The development and history of Benmore has been chronicled extensively (Bown, 1992). This article reviews almost exclusively the timeline regarding the origins and various adaptations of the late 19th-century Walled Garden located towards the northern extremity of the site.

\section{HISTORICAL BACKGROUND}

Walled gardens providing privacy and protection date back to the earliest of recorded civilisations (Goode et al., 1991). In strictly Scottish terms, reliably inclement weather, together with frequently short growing seasons, made the walled garden a necessity to productive gardening (Campbell, 2007). This accepted wisdom was evident as early as 1683 when John Reid (1655-1723) stated in his Scots Gardener regarding the

1. David Gray is a Senior Horticulturist at Benmore Botanic Garden. Address: Benmore Botanic Garden, Dunoon, Argyll, PA23 8QU, UK. Email: dgray@rbge.org.uk 